\title{
Electron Kinetic Effects on Raman Backscatter in Plasmas
}

\author{
M. S. Hur, ${ }^{1}$ R. R. Lindberg, ${ }^{2, *}$ A. E. Charman, ${ }^{2}$ J. S. Wurtele, ${ }^{2,3}$ and H. Suk ${ }^{1, \dagger}$ \\ ${ }^{1}$ Center for Advanced Accelerators, KERI, Changwon, Kyongnam 641-120, Korea \\ ${ }^{2}$ Department of Physics, University of California, Berkeley, Berkeley, California 94720, USA \\ ${ }^{3}$ Center for Beam Physics, Lawrence Berkeley National Laboratory, Berkeley, California 94720, USA
}

(Received 17 March 2005; published 8 September 2005)

\begin{abstract}
We augment the usual three-wave cold-fluid equations governing Raman backscatter (RBS) with a new kinetic thermal correction, proportional to an average of particle kinetic energy weighted by the ponderomotive phase. From closed-form analysis within a homogeneous kinetic three-wave model and ponderomotively averaged kinetic simulations in a more realistic pulsed case, the magnitude of these new contributions is shown to be a measure of the dynamical detuning between the pump laser, seed laser, and Langmuir wave. Saturation of RBS is analyzed, and the role of trapped particles illuminated. Simple estimates show that a small fraction of trapped particles $(\sim 6 \%)$ can significantly suppress backscatter. We discuss the best operating regime of the Raman plasma amplifier to reduce these deleterious kinetic effects.
\end{abstract}

PACS numbers: 52.35.Mw, 42.65.Yj, 52.38.Bv

Electron kinetic effects on stimulated Raman scattering in plasmas have been explored intensively in various contexts, especially in connection with the role of Raman backscatter (RBS) in the ignition phase of inertial confinement fusion $[1,2]$. These investigations were motivated by discrepancies between the results observed in fluid-based simulations and those based on kinetic models. In fully kinetic simulations [1], Vu et al. observed the saturation of Raman reflectivity followed by quasiperiodic bursting. These behaviors have been attributed to a nonlinear phase shift between the three waves associated with trapped particles [1], or to a breakup of the plasma wave by the trapped-particle instability [2].

However, the particle trapping effect described in [1] is more or less phenomenological, in the sense that certain physical terms responsible for the secular phase shift are omitted, and its ultimate dynamical origin remains somewhat unclear. In this Letter, we derive a new kinetic thermal correction from averaging the dynamical equations used in free-electron-laser and averaged-particle-incell (aPIC) models [3]. Its inclusion in the three-wave model provides a clear mathematical encapsulation of electron trapping and other nonlinear effects on RBS saturation and bursting. Analysis indicates that in certain regimes RBS may be saturated predominantly by small levels of electron trapping, rather than by the previously proposed mechanism invoking the breakup of the plasma wave [2]. Our kinetic three-wave model is also useful for the analysis of the Raman backward laser amplifier [3-8], which provided the basis for our study.

We begin with the coupling between pump and seed lasers and the plasma electrons, corresponding to the wave equations implemented in the aPIC computer model [3], used for the kinetic simulations discussed below. Dynamics are derived under a number of simplifying assumptions: viz., field and particle data vary appreciably only in the longitudinal $(z)$ direction; the plasma is under- dense; electron motion remains nonrelativistic; ions remain immobile on the relevant time scales; the seed and pump lasers can be represented as eikonal fields with slowly varying envelopes modulating their respective carrier oscillations, which are at most only weakly detuned from the Raman resonance. With these approximations, the counterpropagating seed and pump laser fields evolve according to

$$
\begin{aligned}
\frac{\partial}{\partial t} a_{s}+c \frac{\partial}{\partial z} a_{s} & =-\frac{i \omega_{p}^{2}}{2 \omega_{1}} a_{p}\left\langle e^{\left.+i \phi_{j}\right\rangle,}\right. \\
\frac{\partial}{\partial t} a_{p}-c \frac{\partial}{\partial z} a_{p} & =-\frac{i \omega_{p}^{2}}{2 \omega_{2}} a_{s}\left\langle e^{-i \phi_{j}}\right\rangle,
\end{aligned}
$$

where $a_{s, p}$ are the eikonal envelopes associated with normalized vector potential of the seed and pump lasers, respectively, in the Coulomb gauge; $\omega_{1,2}$ are the carrier frequencies of the seed and pump, respectively; $\omega_{p}$ is the plasma frequency of the unperturbed plasma of density $n_{0}$. The averaged terms in the right-hand side (RHS) of Eq. (1) are the scaled components of transverse electron current density driving (and driven by) the seed and pump, respectively, normalized by $e n_{0} c$. Canonical momentum conservation was used to derive the averaged current density; the details can be found in [3]. The exponent $\phi_{j} \equiv-k_{b} z_{j}-$ $\Delta \omega t$ is the phase of the $j$ th particle in the beat wave between pump and seed; $\Delta \omega \equiv \omega_{2}-\omega_{1}$ is the beat frequency; and $k_{b} \equiv k_{1}+k_{2}$ is the beat wave number. The angular brackets denote a ponderomotive spatial-averaging operation, namely, $\left\langle Q_{\ell}\right\rangle \equiv \sum_{j: k_{b}\left|z_{j}-z_{\ell}\right| \leq \pi} Q\left(z_{j}, \beta_{j} ; t\right) / N_{0}$ for any observable $Q(z, \beta ; t)$, where $z_{j}(t)$ is the position of the $j$ th particle, $\beta_{j} \equiv \frac{1}{c} \frac{d}{d t} z_{j}$ is its scaled velocity, and the sum extends over all particles within a ponderomotive bucket centered at $z_{\ell}$; and $N_{0} \equiv \frac{2 \pi}{k_{b}} n_{0}^{1 / 3}$ is the initial number of particles in a ponderomotive wavelength. 
We introduce a scaled bunching parameter $F$,

$$
F \equiv-i \frac{\omega_{p}}{\omega}\left\langle e^{-i \phi_{j}}\right\rangle
$$

where $\omega \equiv \frac{1}{2}\left(\omega_{1}+\omega_{2}\right)$ is the average laser frequency, and $\left\langle e^{-i \phi_{j}}\right\rangle$ is a quasilocal characteristic function of the electron spatial distribution function, with remaining spacetime dependence on scales longer and slower than the ponderomotive beat wave. Using the underdense assumption $\omega_{p} \ll \omega_{1,2}$, Eqs. (1) can be transformed into the same form as the laser envelope equations of the cold-fluid threewave model [4]:

$$
\frac{\partial a_{s}}{\partial t}+c \frac{\partial a_{s}}{\partial z}=-\frac{\omega_{p}}{2} a_{p} f^{*}, \quad \frac{\partial a_{p}}{\partial t}-c \frac{\partial a_{p}}{\partial z}=\frac{\omega_{p}}{2} a_{s} f,
$$

where $f$ is the envelope of the Langmuir wave. In the threewave model, $f$ is governed by

$$
\dot{f}+i \delta \omega f=-\frac{\omega}{2} a_{s}^{*} a_{p},
$$

where $\delta \omega \equiv \omega_{p}-\Delta \omega$ represents the detuning from the cold, linear Raman resonance. From the known consistency between the aPIC and fluid three-wave models [3],

$$
\dot{F}+i \delta \omega\left(1+\frac{\delta \omega}{2 \Delta \omega}\right) F+2 \omega \frac{\omega_{p}}{\Delta \omega}\left\langle\beta_{j}^{2} e^{-i \phi_{j}}\right\rangle+\frac{i}{2 \Delta \omega} \ddot{F}
$$$$
-\frac{\omega_{p}^{3}}{2 \omega \Delta \omega} \sum_{\ell=1}^{\ell=\infty} \frac{1}{\ell(\ell+1)}\left\langle e^{-i(\ell+1) \phi_{j}}\right\rangle\left\langle e^{+i \ell \phi_{j}}\right\rangle=-\frac{\omega \omega_{p}}{2 \Delta \omega} a_{s}^{*} a_{p}-\frac{\omega \omega_{p}}{2 \Delta \omega} a_{s} a_{p}^{*}\left\langle e^{-2 i \phi_{j}}\right\rangle .
$$

We next make a number of reasonable ordering assumptions to simplify Eq. (6). Weak detuning implies $\delta \omega \ll$ $\Delta \omega$, while the eikonal approximation has $|\ddot{F}| \ll \omega_{p}|\dot{F}| \ll$ $\omega_{p}^{2}|F|$. Below wave breaking, $|F| \ll 1$, and since each higher harmonic of the plasma wave is excited only by higher-order beating effects, we further expect $\mid\left\langle e^{\left.i \ell \phi_{j}\right\rangle \mid} \sim\right.$ $\left|\left\langle e^{i \phi_{j}}\right\rangle\right|^{\ell}$. With these assumptions, Eq. (6) reduces to

$$
\begin{aligned}
\dot{F}+i \delta \omega F+2 \omega\left\langle\beta_{j}^{2} e^{\left.-i \phi_{j}\right\rangle}\right. & \\
& +\frac{i \omega_{p}}{4}\left\langle e^{-2 i \phi_{j}}\right\rangle F^{*}=-\frac{\omega}{2} a_{s}^{*} a_{p} .
\end{aligned}
$$

Note that Eq. (7) is of the same form as Eq. (4), except for the appearance of the kinetic thermal corrections. The first of these represents modulations in the electron kinetic energy at the beat wave number, which in our case can have contributions from both incoherent thermal motions and the organized oscillation associated with the driven plasma wave. The second measures spatial electron bunching including the leading-harmonic effects of the nonlinear steepening of the plasma-wave profile. In our regime, numerical simulations suggest that this spatial modulation term is negligible compared to the energy modulation term, so we keep only the latter, calling it the kinetic term, although it also includes some purely thermal, i.e., warmfluid, effects.

Because particles trapped in the plasma wave are accelerated to large velocities, we expect the nonlinear contri- the equation of motion for the bunching parameter $F$ is expected to be similar to Eq. (4). To derive the equation of $F$, we start from its first and second time derivatives:

$$
\begin{aligned}
\dot{F}= & i \Delta \omega F+c k_{b} \frac{\omega_{p}}{\omega}\left\langle\beta_{j} e^{-i \phi_{j}}\right\rangle, \\
\ddot{F}= & i \Delta \omega \dot{F}+i \Delta \omega c k_{b} \frac{\omega_{p}}{\omega}\left\langle\beta_{j} e^{-i \phi_{j}}\right\rangle \\
& +i c^{2} k_{b}^{2} \frac{\omega_{p}}{\omega}\left\langle\beta_{j}^{2} e^{-i \phi_{j}}\right\rangle+c k_{b} \frac{\omega_{p}}{\omega}\left\langle\frac{\partial \beta_{j}}{\partial t} e^{-i \phi_{j}}\right\rangle,
\end{aligned}
$$

obtained from Eq. (2). Using the single-particle equation of motion, the last average in the RHS of Eq. ( $5 b$ ) is

$$
\begin{aligned}
\left\langle\frac{\partial \beta_{j}}{\partial t} e^{-i \phi_{j}}\right\rangle= & -\frac{e}{m_{e} c}\left\langle E\left(z_{j}, t\right) e^{-i \phi_{j}}\right\rangle+\frac{i}{4} c k_{b} a_{s}^{*} a_{p} \\
& -\frac{i}{4} c k_{b} a_{s} a_{p}^{*}\left\langle e^{-2 i \phi_{j}}\right\rangle,
\end{aligned}
$$

where $E(z, t)$ is the self-consistent longitudinal electric field, and where the last two terms arise from the ponderomotive force associated with the beating of the seed and pump. Substituting in the harmonic expansion of the electric field, $E(z, t)=\frac{1}{2} \sum_{\ell} E_{\ell} e^{i \ell \phi}+$ c.c., weighting by $e^{-i \phi_{j}}$, and averaging, Eq. (5b) becomes butions from this kinetic term to become significant as more particles are trapped. The effect of this term may be revealed by tracing the relationship between the direction of energy flow and the relative phase and magnitude of the three participating waves. For simplicity, we consider here an infinite, spatially homogeneous system, wherein all spatial derivatives vanish. Decomposition of the complex laser and Langmuir envelopes into their magnitudes and real phases, i.e., as $a_{s, p}=\tilde{a}_{s, p} e^{i \theta_{s, p}}$ and $F=\tilde{F} e^{i \theta_{F}}$, leads to the amplitude equations:

$$
\frac{\partial}{\partial t} \tilde{a}_{s}=-\frac{\omega_{p}}{2} \tilde{a}_{p} \tilde{F} \cos \theta, \quad \frac{\partial}{\partial t} \tilde{a}_{p}=\frac{\omega_{p}}{2} \tilde{a}_{s} \tilde{F} \cos \theta,
$$

and the equation of the relative phase: $\theta \equiv \theta_{s}-\theta_{p}+\theta_{F}$,

$$
\frac{\partial}{\partial t} \theta=K \sin \psi+\Omega-\delta \omega,
$$

where $K$ represents the strength of an effective relativephase "restoring force":

$$
K \equiv \frac{1}{2}\left(\omega_{p} \frac{\tilde{a}_{p} \tilde{F}}{\tilde{a}_{s}}-\omega_{p} \frac{\tilde{a}_{s} \tilde{F}}{\tilde{a}_{p}}+\omega \frac{\tilde{a}_{s} \tilde{a}_{p}}{\tilde{F}}\right),
$$

and $\Omega$ is the effective kinetic thermal detuning:

$$
\Omega \equiv 2 \frac{\omega}{\tilde{F}}\left\langle\beta_{j}^{2} \sin \left(\phi_{j}+\theta_{F}\right)\right\rangle .
$$

Recalling that the driving terms for the magnitude of the laser pump and seed envelopes in Eq. (8) are proportional 
to $\pm \cos \theta$, we can examine how both the relative phase and the energy flow evolve as RBS proceeds. First, we consider a cold three-wave limit, wherein $\Omega$ is neglected. Initially the sign of $K$ is positive, for which the relative phase Eq. (9) has a stable equilibrium at $\theta \simeq \pi$. The driving term for the seed is maximal near this phase, implying that the seed will absorb energy from the pump. However, as the pump is depleted, $K$ will eventually change sign due to the second term on the RHS of Eq. (10). The stable equilibrium point shifts from (nearby) $\pi$ to (nearby) either 0 or $2 \pi$. The relative phase $\theta$ will move to one of the new stable equilibria, at which point the seed's driving term $(\propto-\cos \theta)$ becomes negative, and the seed begins to return energy to the pump. Figure 1 shows plots of the laser amplitudes, $K$, and $(-\cos \theta)$ from an initial-value numerical solution of the fluid three-wave model Eqs. (3) and (4). Those quantities are represented as functions of spatial position, which roughly corresponds to evolved time in the spatially homogeneous case. As the pump is depleted, $K$ becomes negative, and $\theta$ rapidly approaches the new equilibrium. Then the $(-\cos \theta)$ term again becomes negative, and the seed and plasma wave begin to give energy back to the pump, resulting in the appearance of the secondary bump in the pump profile, seen experimentally as a quasiperiodic bursting in the RBS reflectivity.

It is clear that in the cold-fluid three-wave model, the change in the direction of energy flow is driven predominantly by pump depletion. We will show that, in the kinetic three-wave model, particle trapping is more important than pump depletion in changing the direction of energy flow, and suppression of RBS can occur long before significant pump depletion [compare Figs. 1(a) and 2(a)]. Before the onset of particle trapping, the kinetic term $\Omega$ remains relatively small and does not change the physical behavior qualitatively from that observed in the cold-fluid model. As particles are trapped and accelerated in the plasma-wave potential, the magnitude of the kinetic detuning increases, causing the stability of the phase equilibria to decrease.

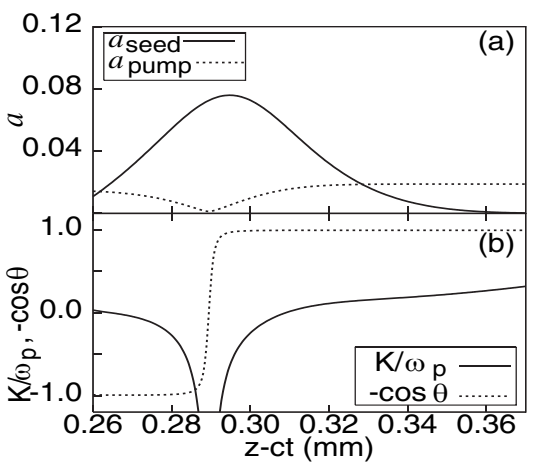

FIG. 1. (a) The envelopes of the seed and pump calculated numerically from the cold three-wave model and (b) corresponding $K$ and $(-\cos \theta)$. We have used $a_{s}=0.0006, \lambda_{s}=$ $0.873 \mu \mathrm{m}$ for the seed, $a_{p}=0.0188, \lambda_{p}=0.8 \mu \mathrm{m}$ for the pump, and the plasma density $1 \times 10^{19} \mathrm{~cm}^{-3}$. The snapshot was captured at $t=2.1 \mathrm{ps}$.
When the absolute value of the total detuning $\delta_{\text {tot }} \equiv$ $-\delta \omega+\Omega$ exceeds $|K|$, the RHS of Eq. (9) is shifted positive or negative according to the sign of $\delta_{\text {tot }}$, and the equilibria vanish altogether. The relative phase $\theta$ then begins to roll over in one direction or the other, at which time the direction of the energy flow changes its sign. This process is visualized in Fig. 2, obtained from a fully kinetic aPIC simulation. In this aPIC code, the envelope Eqs. (1) are used to evolve the lasers, while the electrons are modeled using conventional particle-in-cell methods. The total detuning $\delta_{\text {tot }}, K$, and the relative phase $\theta$ were calculated from the simulation data. At $z \sim 0.34 \mathrm{~mm}$ significant particle trapping begins, and $\Omega$ starts to increase. The total detuning begins to outgrow $K$ at $z \sim 0.33 \mathrm{~mm}$, and the driving term $(-\cos \theta)$ starts to decrease rapidly at this point, finally flipping its sign. Note that during this process, the sign of $K$ remains positive, a pronounced difference from the cold three-wave model. After $z \sim 0.28 \mathrm{~mm} \cos \theta$ oscillates rapidly between \pm 1 in a stochastic-looking manner, suggesting net energy exchange will be negligible beyond that point. The extent of trapping is revealed in Fig. 3, which shows electron phase-space portraits at three different places: Fig. 3(a) shows a beat-wave bucket near $z \sim 0.36 \mathrm{~mm}$ where there is no trapping, and therefore $\Omega$ remains small. Significant electron trapping is shown in Fig. 3(b), which is the phase portrait near the peak of the seed $(z \sim 0.33 \mathrm{~mm})$. The total detuning is larger than $K$ in this region. Figure 3(c) lies in the stochastic region, where $\cos \theta$ shows rapid, noisy oscillations. The particle phasespace appears chaotic, which is characteristic of nonlinear wave breaking. Figure 3(d) represents the electron velocity distributions for Figs. 3(a) and 3(b). The flattening in the left-side tail indicates the trapping.

The simulation indicates that about $6 \%$ of the particles are trapped when the seed reaches its peak value. This was measured from Fig. 3(d) by dividing the area under the flat tail by the total area under the distribution curve (solid line). To understand how such a small fraction of trapped particles can change the system behavior so dramatically,

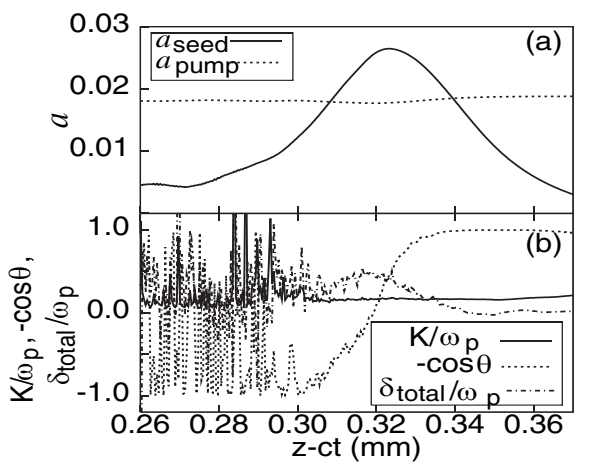

FIG. 2. (a) The envelopes of the seed and pump from an $a P I C$ simulation and (b) corresponding $K,(-\cos \theta)$, and $\delta_{\text {tot }}$. Parameters and simulation time were the same as used in Fig. 1, and the plasma temperature was $40 \mathrm{eV}$, for which Landau damping is negligible. 


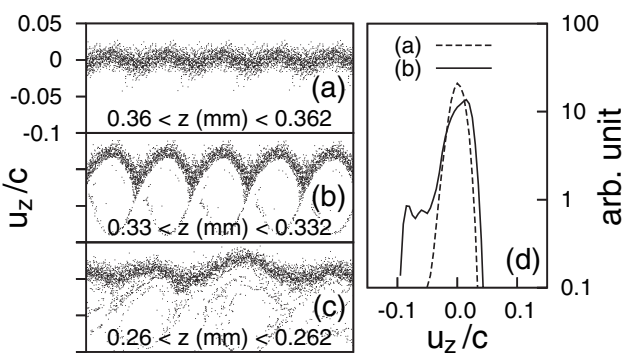

FIG. 3. Simulated particle phase space observed at three different positions: (a) no trapping ahead of the seed, (b) trapping near the peak of the seed, and (c) detrapped particles and plasma behind the seed. (d) Corresponding velocity distributions for (a) (dashed line) and (b) (solid line).

we estimate the ratio $|\Omega / K|$. The average velocity of trapped particles is given by the phase velocity of the plasma wave, $\beta_{p}=\Delta \omega / k_{b}$. Furthermore, the trapped electrons are no longer spread over the entire bucket phase, as the turning points having been moved in by the angle $\psi_{c}$ due to their slow crossing of the separatrix. Using these definitions, we see that for a trapped fraction $\eta$, the kinetic detuning (11) will scale as $\Omega \sim 4 \eta \sin \left(\psi_{c}\right) \omega \beta_{p}^{2} / \tilde{F}$. Approximate analytic expressions for the parameters $\eta$ and $\psi_{c}$ may be derived based on adiabatic separatrix crossing theory [9], but here we just estimate them directly from the simulations. To evaluate $K$, observe that the last term in the definition (10) is the dominant one (since $\omega \gg \omega_{p}$ ), so that $K \sim 2 \omega \tilde{a}_{s} \tilde{a}_{p} / \tilde{F}$. Using these approximations, we find

$$
\left|\frac{\Omega}{K}\right| \sim 8 \eta\left|\sin \psi_{c}\right| \frac{\beta_{p}^{2}}{\tilde{a}_{s} \tilde{a}_{p}} .
$$

From Fig. 3(b) we measure a trapped fraction of $\eta \approx 6 \%$ and an angle $\psi_{c} \approx \pi / 8$, while Fig. 2(a) has $\tilde{a}_{s} \sim \tilde{a}_{p} \sim$ 0.02 . Using these parameters and $\beta_{p} \approx .04$, the ratio $|\Omega / K|$ is $O(1)$, which is the threshold for the disappearance of the equilibria of Eq. (9).

As trapped electrons are accelerated in the plasma-wave potential, the plasma wave loses energy to compensate, and undergoes a frequency downshift. This process is readily seen from Eq. (9) and the phase equation of the plasma wave: $\frac{\partial}{\partial t} \theta_{F} \simeq K \sin \theta-\delta \omega+\Omega$. As trapping occurs, the kinetic detuning $\Omega$ outruns the driving term. Hence, $\frac{\partial}{\partial t} \theta_{F}$ becomes positive, which corresponds to a downshift in the plasma-wave frequency. Specifically, for an undriven plasma wave, where $K=0$, the phase equation for the plasma wave becomes $\frac{\partial}{\partial t} \theta_{F}=\Omega$. Therefore the kinetic thermal detuning term can be thought as a measure of the nonlinear frequency shift due to trapping, or to any other nonlinear kinetic or fluidlike effects.

We now consider these kinetic effects in the context of the Raman backward amplifier. Raman amplification has been proposed to augment chirped-pulse-amplification techniques by raising the intensity achievable for ultrashort laser pulses. While various constraints on operating regimes have been identified [8], kinetic effects, which may impose additional fundamental restrictions, have received relatively little attention. From the estimates in $[8,10,11]$, driving the amplifier below the trapping or wave-breaking limit requires that the pump intensity be less than $2 \times 10^{14} \mathrm{~W} / \mathrm{cm}^{2}$. In order to compress such a pump to intensities of $10^{18} \mathrm{~W} / \mathrm{cm}^{2}$, the interaction length must exceed a few centimeters, which presents a formidable challenge. The analysis of the kinetic term suggests a means to reduce deleterious trapping effects in the Raman backward amplifier. In this case, the peak plasma-wave amplitude can be shown to be $O\left(a_{p} \sqrt{\omega / \omega_{p}}\right)$ [8]. After a little algebra, the ratio (12) can then be represented as a nearly explicit function of the pump and seed amplitudes: $\left|\frac{\Omega}{K}\right| \sim \frac{8 \eta\left|\sin \psi_{c}\right| \beta_{p}}{\tilde{a}_{s}}\left[2\left(\frac{4 \omega}{\omega_{p}}\right)^{1 / 4}+\left(\frac{\beta_{p}}{\tilde{a}_{p}}\right)\right]^{2}$. Even in the strong trapping regime, where $\eta$ is relatively large, starting with a sufficiently strong seed may enable the ratio $|\Omega / K|$ to remain smaller than unity, whereby kinetic detuning prior to pump depletion can be avoided. More intensive simulations designed to investigate these processes are in progress.

In conclusion, we derived a new kinetic thermal term to be incorporated in the three-wave model, proportional to an average of particle kinetic energy weighted by each particle's ponderomotive phase. From an analysis with this kinetic three-wave model, it is shown that at least in certain parameter regimes, electron trapping saturates Raman reflectivity prior to the breakup of the plasma wave. Furthermore, we have revealed a physical term responsible for the trapping-induced secular phase shift. For the case of Raman amplifier, the deleterious trapping effect can be reduced by operating the system with a sufficiently strong initial seed pulse.

We acknowledge financial support from the Division of High Energy Physics, U.S. Department of Energy, from DARPA, U.S. Department of Defense, and from Creative Research Initiatives, Republic of Korea.

*Electronic address: RL236@socrates.berkeley.edu ${ }^{\dagger}$ Electronic address: hysuk@keri.re.kr

[1] H.X. Vu, D.F. DuBois, and B. Bezzerides, Phys. Rev. Lett. 86, 4306 (2001); Phys. Plasmas 9, 1745 (2002).

[2] S. Brunner and E. J. Valeo, Phys. Rev. Lett. 93, 145003 (2004).

[3] M. S. Hur et al., Phys. Plasmas 11, 5204 (2004).

[4] V. M. Malkin, G. Shvets, and N. J. Fisch, Phys. Rev. Lett. 82, 4448 (1999); Phys. Plasmas 7, 2232 (2000).

[5] P. Mardahl et al., Phys. Lett. A 296, 109 (2002).

[6] J. Kim et al., Phys. Lett. A 314, 464 (2003).

[7] W. Cheng et al., Phys. Rev. Lett. 94, 045003 (2005).

[8] D. S. Clark and N. J. Fisch, Phys. Plasmas 10, 3363 (2003).

[9] R. R. Lindberg, A. E. Charman, and J.S. Wurtele (to be published).

[10] W. L. Kruer, The Physics of Laser Plasma Interactions (Addison-Wesley, New York, 1988).

[11] T. P. Coffey, Phys. Fluids 14, 1402 (1971). 